\title{
Accidental degeneracy and hidden symmetry: Rectangular wells with commensurate sides
}

\author{
R. Lemus \\ Instituto de Ciencias Nucleares, UNAM, Apdo. Postal 70-543, Circuito Exterior, C.U. 04510 Mexico, D.F., \\ Mexico

\begin{abstract}
A. Frank
Instituto de Ciencias Nucleares, UNAM, Apdo. Postal 70-543, Circuito Exterior, C.U. 04510 Mexico, D.F., Mexico and Instituto de Física, Laboratorio de Cuernavaca, UNAM, Apdo. Postal 139-B, Cuernavaca, Morelos, Mexico
\end{abstract}

\section{V. Andrés} \\ Departamento de Física Atómica, Molecular y Nuclear Facultad de Física, Universidad de Sevilla, \\ Apdo. 1065, 41080 Sevilla, Spain \\ F. Leyvraz \\ Instituto de Física, Laboratorio de Cuernavaca, UNAM, Apdo. Postal 139-B, Cuernavaca, Morelos, Mexico
}

(Received 28 July 1997; accepted 26 November 1997)

\begin{abstract}
Two-dimensional quantum square wells and rectangular billiards with commensurate sides are simple systems which exhibit accidental degeneracies. We show that a recent analysis for square wells can be similarly applied to rectangular wells with commensurate sides. (C) 1998 American Association of Physics Teachers.
\end{abstract}

\section{INTRODUCTION}

In a recent paper we have shown that the impenetrable square-well potential exhibits accidental degeneracy and that it can be understood in terms of a hidden "dynamical symmetry." 1 The fact that part of the double degeneracies in this system is not explained by the patent geometrical symmetry of the square box is not widely appreciated, ${ }^{2}$ since double degeneracies are expected for the $\mathscr{C}_{4 v}$ symmetry group of the square box. In other words, the unexpected result is that some of the doublets do not transform as the two-dimensional representation " $E$ "' of $\mathscr{C}_{4 v}$, and thus the continuous symmetry of Ref. 1 has to be invoked in order to construct a larger symmetry group. ${ }^{1}$

The case of impenetrable rectangular wells is a more intriguing example of accidental degeneracy, which occurs whenever the rectangle's sides are commensurate, since in this situation no geometrical transformation can be found that transforms the degenerate states into each other. From a more technical point of view, the patent symmetry group of a rectangular box has only one-dimensional representations. ${ }^{3}$

How can we understand this peculiar result? In this article we shall show that rectangular boxes with commensurate sides possess a discrete hidden symmetry that leads to a remarkable analogy between its states and those of the square box, from the point of view of symmetry. We shall thus be able to relate the accidental degeneracy of the former to our previous work on the latter. ${ }^{1}$

\section{COMMENSURATE RECTANGULAR-WELL POTENTIAL}

A free particle enclosed by an impenetrable twodimensional rectangular well of sides $L_{1}$ and $L_{2}$ has the eigenstates

$$
\psi_{n_{1} n_{2}}(x, y)=\frac{2}{\sqrt{L_{1} L_{2}}} \sin \frac{n_{1} \pi x}{L_{1}} \sin \frac{n_{2} \pi y}{L_{2}},
$$

where $n_{1}, n_{2}$ are positive integers. These states satisfy the condition $\psi_{n_{1} n_{2}}=0$ at the boundaries of the box, when the origin of the coordinate system is chosen at the lower left corner, as shown in Fig. 1.

The system's Hamiltonian

$$
H=-\frac{\hbar^{2}}{2 m}\left(\frac{\partial^{2}}{\partial x^{2}}+\frac{\partial^{2}}{\partial y^{2}}\right)
$$

gives rise to the eigenvalues

$$
E_{n_{1}, n_{2}}=\frac{\pi^{2} \hbar^{2}}{2 \mu}\left[\frac{n_{1}^{2}}{L_{1}^{2}}+\frac{n_{2}^{2}}{L_{2}^{2}}\right],
$$

which are in general nondegenerate. For commensurate side lengths, however, i.e.,

$$
n L_{1}=m L_{2} \equiv L_{0}, \quad n, m \text { integer, }
$$

we find

$$
E_{n_{1} n_{2}}=\frac{\pi^{2} \hbar^{2}}{2 \mu L_{0}^{2}}\left[n^{2} n_{1}^{2}+m^{2} n_{2}^{2}\right] .
$$

It is then simple to see that the states $\psi_{n_{1}, n_{2}}$ and $\psi_{n_{1}^{\prime} n_{2}^{\prime}}$ are degenerate if the following conditions are satisfied:

$$
n n_{1}=m n_{2}^{\prime}, \quad m n_{2}=n n_{1}^{\prime} .
$$

Given $n$ and $m$, Eqs. (6) will be satisfied only for a subset of all eigenvalues (3). As in the square-box case, additional degeneracies are found for "Pythagorean" identities such as

$$
\left(n n_{1}\right)^{2}+\left(m n_{2}\right)^{2}=\left(n n_{3}\right)^{2}+\left(m n_{4}\right)^{2},
$$

which we shall not consider further in our discussion. ${ }^{1}$

The double degeneracies $E_{n_{1}, n_{2}}=E_{n_{1}^{\prime}, n_{2}^{\prime}}$, occurring when $\left(n_{1}, n_{2}\right)$ and $\left(n_{1}^{\prime}, n_{2}^{\prime}\right)$ satisfy conditions (6) [and $\left(n_{1}^{\prime}, n_{2}^{\prime}\right)$ $\left.\neq\left(n_{1}, n_{2}\right)\right]$, are the ones we would like to explain from the point of view of symmetry. 


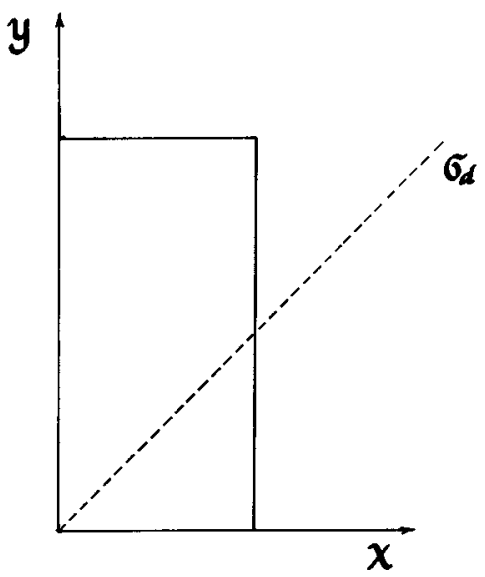

Fig. 1. Selected coordinate system for the rectangular-well potential.

The patent symmetry group of the rectangular box, with or without commensurate sides, is the group $\mathscr{C}_{2 v}$, whose structure is depicted in Fig. 2. It consists of the two reflections $\hat{\sigma}_{v}^{a}$ and $\hat{\sigma}_{v}^{b}$, the rotation through $\pi$ about the $z$ axis, and the identity $E$. The $\mathscr{C}_{2 v}$ character table is shown in Table I. Since all the operations of $\mathscr{C}_{2 v}$ commute, the group is Abelian, which in turn means that all its irreducible representations (irreps) are one dimensional, ${ }^{3}$ as mentioned in the Introduction. The eigenfunctions (1) span irreducible representations of the symmetry group $\mathscr{C}_{2 v}$ in accordance with the following:

\begin{tabular}{cll} 
& \multicolumn{2}{c}{ Parity } \\
\cline { 2 - 3 } Irrep label & $n_{1}$ & $n_{2}$ \\
\hline$A_{1}$ & odd & odd \\
$A_{2}$ & even & even \\
$B_{1}$ & even & odd \\
$B_{2}$ & odd & even
\end{tabular}

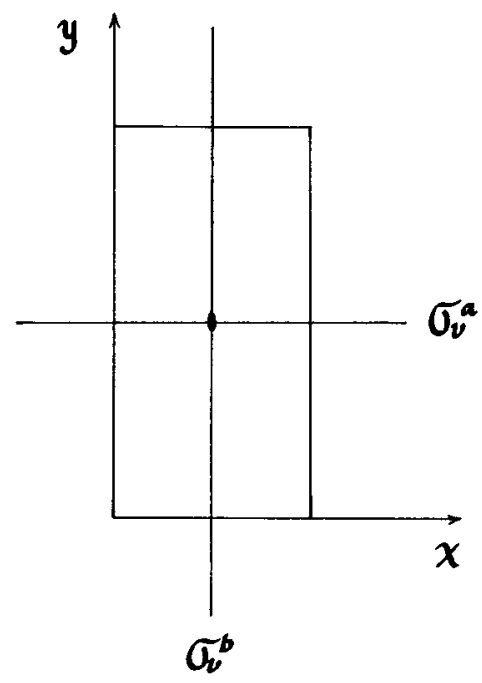

Fig. 2. Symmetry elements of the group $\mathscr{C}_{2 v}$.
Table I. Character table of the group $\mathscr{C}_{2 v}$.

\begin{tabular}{lrrrrrl}
\hline \hline $\mathscr{C}_{2 v}$ & $E$ & $C_{2}$ & $\sigma_{v}^{a}$ & $\sigma_{v}^{b}$ & & \\
\hline$A_{1}$ & 1 & 1 & 1 & 1 & $z$, & $x^{2}, y^{2}$ \\
$A_{2}$ & 1 & 1 & -1 & -1 & & $x y$ \\
$B_{1}$ & 1 & -1 & 1 & -1 & $x$, & $x z$ \\
$B_{2}$ & 1 & -1 & -1 & 1 & $y$, & $y z$ \\
\hline \hline
\end{tabular}

as can be readily deduced from the action of the symmetry transformations $R \in \mathscr{C}_{2 v}$ on the eigenstates (1). From the point of view of the patent symmetry of the rectangular box there is no reason for the double degeneracy encountered. In the next section we find a "hidden" symmetry operation that leads to an explanation of this matter.

\section{HIDDEN SYMMETRY}

When we substitute conditions (6) into the eigenstates (1) and use the relation (4) between the side lengths, we find

$$
\begin{aligned}
\psi_{n_{1}^{\prime} n_{2}^{\prime}}(x, y) & =\frac{2}{\sqrt{L_{1} L_{2}}} \sin \frac{n_{1}^{\prime} \pi x}{L_{1}} \sin \frac{n_{2}^{\prime} \pi y}{L_{2}} \\
& =\frac{2}{\sqrt{L_{1} L_{2}}} \sin \frac{m n_{2} \pi x}{n L_{1}} \sin \frac{n n_{1} \pi y}{m L_{2}} \\
& =\frac{2}{\sqrt{L_{1} L_{2}}} \sin \frac{n_{2} \pi x}{L_{2}} \sin \frac{n_{1} \pi y}{L_{1}}=\psi_{n_{1}, n_{2}}(y, x) .
\end{aligned}
$$

Equation (9) leads to the remarkable result that when $\psi_{n_{1}^{\prime} n_{2}^{\prime}}(x, y)$ is degenerate to $\psi_{n_{1} n_{2}}(x, y)$, it follows that

$$
\psi_{n_{1}^{\prime} n_{2}^{\prime}}(x, y)=\hat{\sigma}_{d}^{a} \hat{\psi}_{n_{1} n_{2}}(x, y),
$$

where we have used the notation of Ref. 1 and indicated by $\hat{\sigma}_{d}^{a}$ the operation that interchanges the $x$ and $y$ coordinates in the wave functions. As is clear from Fig. 1, the $\hat{\sigma}_{d}^{a}$ operation is not a patent (geometric) symmetry of the rectangular box, but rather a hidden (dynamic) symmetry of the system.

The problem is not yet fully solved, however, since, as explained in Ref. 1, not all the cases of double degeneracy can be explained by this symmetry. To illustrate this statement we shall consider the case $n=2, m=1$ in (4). The spectrum of this rectangular box is shown in Fig. 3 up to the first 12 levels. The states are characterized by the quantum numbers $\left(n_{1} n_{2}\right)$ and the symmetry label of $\mathscr{C}_{2 v}$. The states fulfilling the conditions (6) are indicated in boldface $\left(\mathbf{n}_{1}, \mathbf{n}_{2}\right)$. Note that some states satisfy $\left(n_{1} n_{2}\right)=\left(n_{1}^{\prime} n_{2}^{\prime}\right)$, so that no degeneracy appears.

We now turn our attention to the doubly degenerate states. In order to identify the cases where the operator $\hat{\sigma}_{d}^{a}$ explains the degeneracy, it is convenient to construct a representation of the operators. We first consider the doublet $\left(\psi_{14}^{B_{2}}, \psi_{22}^{A_{2}}\right)$, which defines a subspace spanning the following representation for the operators associated with $\mathscr{C}_{2 v}$ and the dynamical operator $\hat{\sigma}_{d}^{a}$ : 


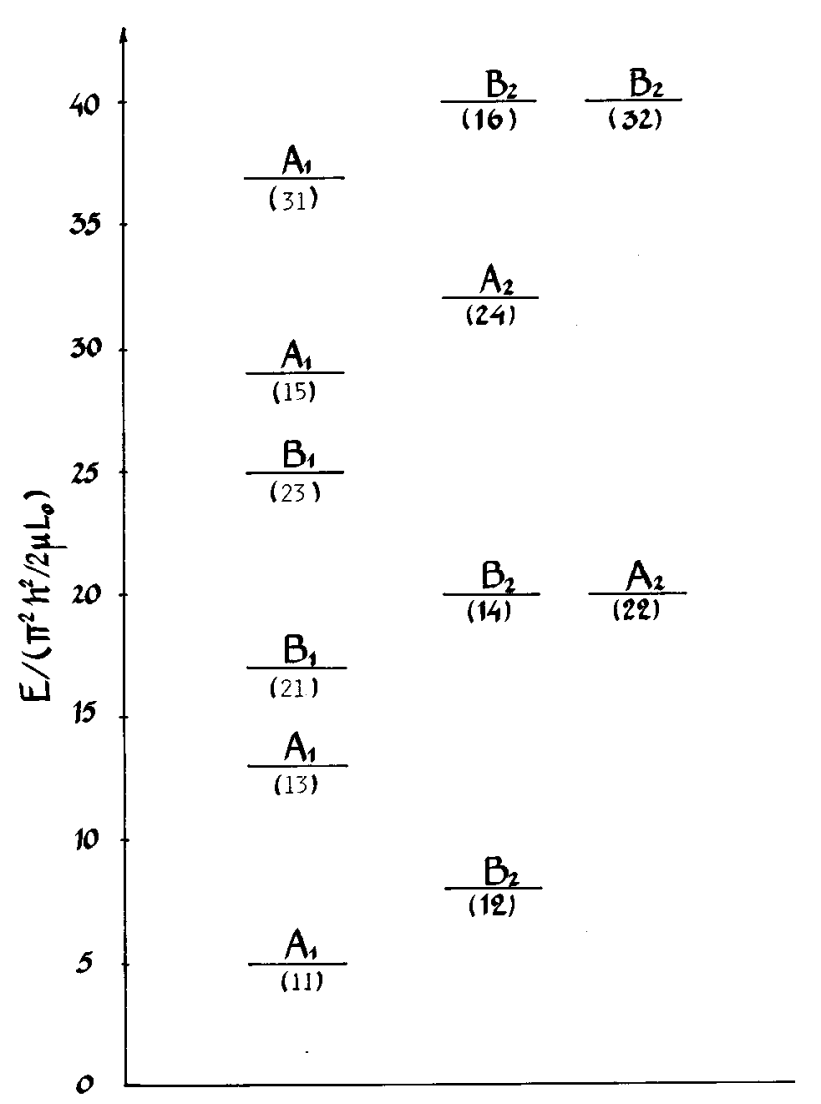

Fig. 3. Spectrum associated with a rectangular box for $n=2$ and $m=1$ in Eq. (4).

$$
\begin{array}{ll}
\mathbf{D}\left(C_{2}\right) & =\left(\begin{array}{cc}
1 & 0 \\
0 & -1
\end{array}\right), \quad \mathbf{D}\left(\sigma_{v}^{a}\right)=\left(\begin{array}{cc}
-1 & 0 \\
0 & -1
\end{array}\right), \\
\mathbf{D}\left(\hat{\sigma}_{d}^{a}\right)=\left(\begin{array}{ll}
0 & 1 \\
1 & 0
\end{array}\right), &
\end{array}
$$

where we only need to specify the generators of $\mathscr{C}_{2 v}$. From the form of the representations (11) one can show that it is not possible to diagonalize simultaneously these matrices. This means that the representation (11) is irreducible and consequently that the operator $\hat{\sigma}_{d}^{a}$ can be identified as the hidden symmetry that explains the degeneracy. It is necessary, however, to define the new functions

$$
\psi_{1}=\psi_{22}^{A_{2}}+\psi_{14}^{B_{2}}, \quad \psi_{2}=\psi_{22}^{A_{2}}-\psi_{14}^{B_{2}},
$$

since they are the ones that carry the labels corresponding to the full symmetry. ${ }^{1}$

On the other hand, the representation generated by the functions $\left(\psi_{16}^{B_{2}} \psi_{32}^{B_{2}}\right)$ is reducible, as can be shown from its explicit form

$$
\begin{array}{ll}
\mathbf{D}\left(C_{2}\right)=\left(\begin{array}{cc}
-1 & 0 \\
0 & -1
\end{array}\right), & \\
\mathbf{D}\left(\sigma_{v}^{a}\right)=\left(\begin{array}{cc}
-1 & 0 \\
0 & -1
\end{array}\right), \quad \mathbf{D}\left(\sigma_{d}\right)=\left(\begin{array}{ll}
0 & 1 \\
1 & 0
\end{array}\right) .
\end{array}
$$

The change of basis

$$
\phi_{1}=\psi_{16}^{B_{2}}+\psi_{32}^{B_{2}}, \quad \phi_{2}=\psi_{16}^{B_{2}}-\psi_{32}^{B_{2}},
$$

diagonalizes the representation (13). This will happen whenever the partners $\left(\psi_{n_{1} n_{2}}^{\Gamma}, \psi_{n_{1}^{\prime} n_{2}^{\prime}}^{\Gamma}\right)$ carry the same irreducible representation $\Gamma$ of $\mathscr{C}_{2 v}$. It follows that the operator $\hat{\sigma}_{d}^{a}$ cannot explain the degeneracy for all cases. It turns out, however, that in complete analogy to the square-box system, ${ }^{1}$ the additional degeneracies can be explained by the introduction of the integral of motion

$$
\hat{D}^{\left(A_{1}\right)}=\frac{\partial^{2}}{\partial x^{2}}-\frac{\partial^{2}}{\partial y^{2}},
$$

where $\left(A_{1}\right)$ indicates the $\mathscr{C}_{2 v}$ character of the operator. This $\mathscr{C}_{2 v}$ symmetry implies that the operator satisfies the requirement that it can connect the wave functions carrying the same $\mathscr{C}_{2 v}$ irreducible representation. In fact, the action of $D^{\left(A_{1}\right)}$ over the space (14) can be easily shown to be given by the matrix

$$
\left\|\left\langle\phi_{i}\left|\hat{D}^{A_{1}}\right| \phi_{j}\right\rangle\right\|=\left(\begin{array}{ll}
0 & 1 \\
1 & 0
\end{array}\right), \quad i, j=1,2,
$$

which means that the operator (15) interchanges the degenerate states and thus represents the additional hidden symmetry necessary to explain the degeneracy of the wave functions $\left(\psi_{n_{1} n_{2}}^{\Gamma}, \psi_{n_{1}^{\prime} n_{2}^{\prime}}^{\Gamma}\right)$.

We have found two operators, namely $\hat{\sigma}_{d}^{a}$ and $\hat{D}^{\left(A_{1}\right)}$, which represent hidden symmetries of the rectangular box with commensurate sides. These operators, together with $\mathscr{C}_{2 v}$, generate a group which, however, is not the same as the one appearing in the square-well system. This is a consequence of the fact that in this case the symmetry element $\hat{\sigma}_{d}^{a}$ and the $\mathscr{C}_{2 v}$ group elements do not intersect, as can be seen in Fig. 2. The discussion of the group generated by these operators is beyond the scope of this paper. We only wish to mention that such group corresponds to the planar space group ${ }^{4} p 4 m m$, which together with the integral of motion $\hat{D}^{\left(A_{1}\right)}$ gives rise to the group $D(1) \wedge p 4 m m$, which is analogous to the group $D(1) \wedge \mathscr{C}_{4 v}$ appearing in the squarebox problem. ${ }^{1}$

\section{CONCLUSIONS}

In this paper we have extended our previous analysis of accidental degeneracy in the square-box system to the case of rectangular wells with commensurate side lengths. Our main results are contained in Eqs. (9) and (10), which indicate that the subset of states in the latter system which satisfy relations (6) are closely linked to the states in the former from the point of view of symmetry. In this respect, the rectangular-well's accidental degeneracy and its explanation in terms of a connection to the square-box system perhaps represent a more subtle and interesting result than that found for the square box itself, where part of the degeneracy can be explained by the patent $\mathscr{C}_{4 v}$ symmetry. ${ }^{1}$

${ }^{1}$ F. Leyvraz, A. Frank, R. Lemus, and M. Andrés “Accidental degeneracy in a simple quantum system: A new symmetry group for a particle in an impenetrable square well potential,' Am. J. Phys. 65 (11), 1087-1094 (1997).

${ }^{2}$ Claude Cohen-Tannoudji, Bernard Dui, and Frank Laloë, Quantum Mechanics (Wiley, New York, 1977).

${ }^{3}$ Morton Hammermesh, Group Theory and Its Application to Physical Problems (Dover, New York, 1989).

${ }^{4}$ Gerald Burns and A. M. Glazer, Space Groups for Solid State Scientists (Academic, New York, 1990). 